\title{
CHIARITÉ
}

\section{Emigration von Sozialmedizinern der Berliner Charité in die USA - Karrieren der Schüler Alfred Grotjahns}

S. N. Willich, K. Etzold, A. Berghöfer

\section{Document type}

Postprint (accepted version)

This version is available at

https://doi.org/10.17169/refubium-32508

\section{Citation details}

Willich S, Etzold K, Berghöfer A. Emigration von Sozialmedizinern der Berliner Charité in die USA Karrieren der Schüler Alfred Grotjahns. Das Gesundheitswesen. Georg Thieme Verlag KG; 2007. p. 694-698. DOI: 10.1055/s-2007-993230

\section{Terms of use}

All rights reserved. This document is intended solely for personal, non-commercial use. 


\title{
Emigration von Sozialmedizinern der Berliner Charité in die USA - Karrieren der Schüler Alfred Grotjahns ${ }^{1}$
}

\author{
Stefan N. Willich, Kristin Etzold, Anne Berghöfer
}

Institut für Sozialmedizin, Epidemiologie und Gesundheitsökonomie, Charité - Universitätsmedizin Berlin

Korrespondenzadresse:

Prof. Dr. med. Stefan N. Willich, MPH

Institut für Sozialmedizin, Epidemiologie und Gesundheitsökonomie

Charité - Universitätsmedizin Berlin

10098 Berlin

Tel.: 030/ 450529002

FAX: 030/ 450529902

email: stefan.willich@charite.de

1 Der Beitrag ist Teil der Dissertation „Exodus der Sozialmedizin in den dreißiger Jahren von Berlin in die USA - das Erbe Alfred Grotjahns". Charité Universitätsmedizin Berlin, 2007 


\section{Zusammenfassung}

Hintergrund:

Der Arzt Alfred Grotjahn wurde 1920 an der Universität Berlin auf den ersten Lehrstuhl für Sozialhygiene in Deutschland berufen. In der Zeit des Nationalsozialismus wurde das Institut für Sozialhygiene geschlossen bzw. von 1933 bis 1945 in ein Institut für Rassenhygiene umgewandelt. In dieser Zeit emigrierten viele deutsche Wissenschaftler aufgrund politischer, religiöser oder rassischer Verfolgung, so auch Sozialmediziner der Berliner Charité.

Methodik:

Basis dieser biographischen Darstellung von Schülern Grotjahns, die in die USA emigrierten, sind Nachforschungen in deutschen sowie amerikanischen Quellen, u.a. Archiv der Humboldt-Universität Berlin, Bundesarchiv in Koblenz, National Institute of Health in Washington, NARA-Archive in Washington und Alan-Mason-ChesneyMedical-Archives in Baltimore.

Ergebnisse:

Die jüdischen Wissenschaftler Franz Goldmann, Miron Kantorowicz, Alfred Korach und Georg Wollf, ehemalige Mitarbeiter von Alfred Grotjahn, mussten das Land in den 30er Jahren verlassen und emigrierten in die USA. Sie waren in unterschiedlichen Public Health Bereichen tätig und hatten relativ erfolgreiche Karrieren.

Diskussion: Gesellschaftssystem und Mentalität der USA haben den beruflichen Hintergrund und die Emigrationserfahrungen ehemaliger Sozialmediziner der Charité offenbar positiv gewertet.

Schlüsselwörter: Sozialmedizin, Sozialhygiene, Rassenhygiene, Nationalsozialismus, Emigration, Public Health. 


\section{Abstract}

Background:

In 1920, the physician Alfred Grotjahn was appointed as first chair for social hygiene in Germany at the University of Berlin. During national socialism, the institute for social hygiene was closed and from 1933 and 1945 transformed into an institute for racial hygiene. During that period, many German scientists including social medicine coworkers of the Berlin Charite emigrated due to political, religious or racial prosecution.

Methods:

The basis for this biographical presentation of coworkers of Grotjahn, who emigrated into the US, is based on research conducted at German and American sources Archive of Humboldt University of Berlin, Federal Archive in Koblenz, National Institutes of Health in Washington, NARA Archive in Washington, and Alan-Mason-ChesneyMedical-Archives in Baltimore.

Results:

The Jewish scientists Franz Goldmann, Miron Kantorowicz, Alfred Korach and Georg Wollf, all former coworkers of Alfred Grotjahn, were forced to leave the country in the 30ies and emigrated to the USA. They were active in different areas of public health and pursued relatively successful careers.

Discussion: The societal system and mentality of the US have obviously valued the professional background and experience of emigration of former social medicine experts of the Charité.

Key words:

Social medicine, social hygiene, racial hygiene, national socialism, emigration, public health 


\section{Einleitung und Methodik}

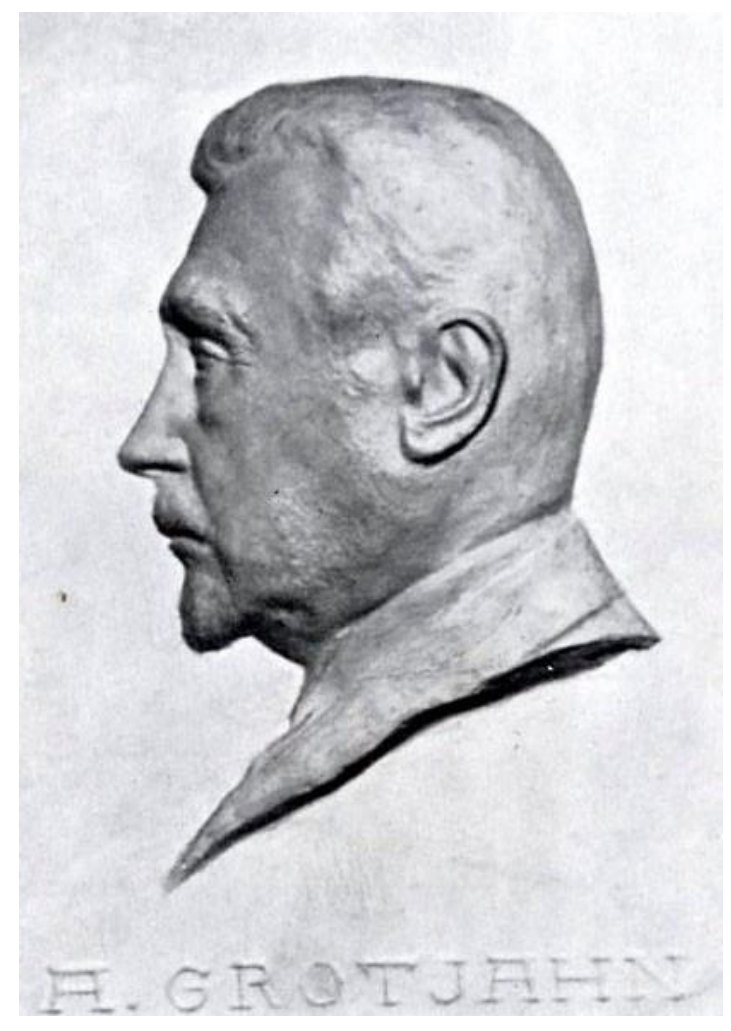

Abb. 1: Alfred Grotjahn; Institut für Sozialmedizin, Epidemiologie und Gesundheitsökonomie der Charité

Mit steigendem Wissensstand um soziale Prädiktoren von Krankheiten entwickelte sich gegen Ende des 19. Jahrhunderts aus der bereits eigenständigen Wissenschaft der "Hygiene" die "Sozialhygiene" (1). Zeitgleich entstand auch der Begriff "Soziale Medizin". Das eigenständige Profil erhielt dieses Fach durch Alfred Grotjahn (2). Grotjahn, 1869 als Sohn einer niedersächsischen Arztfamilie geboren, studierte von 1890-1896 Medizin an den Universitäten Greifswald, Leipzig, Kiel und Berlin, und arbeitete zunächst als niedergelassener, praktischer Arzt in Berlin. Hier sowie auf Reisen nach London und Paris 1902 gewann er Eindrücke von der gesundheitlichen Lage der Großstadtbevölkerung. Grotjahn verstand als Ziel der Sozialhygiene vor allem die Prävention von Krankheiten. Er integrierte hierbei das damals populäre Gedankengut der praktischen Eugenik in sein Konzept einer bevölkerungspolitischen Anwendung der Prävention (3). 1912 wurde Grotjahn Privatdozent für Hygiene und Leiter der Abteilung für Sozialhygiene am Hygiene-Institut der Universität in Berlin. 1920 erlangte er trotz des Widerstandes der überwiegenden Mehrheit des Fakultätsrates, die ein Ordinariat für Sozialhygiene für überflüssig hielten, die Professorenwürde und war damit der erste und damals einzige Ordinarius für Sozialhygiene in Deutschland $(4,5)$.

Der Nationalsozialismus unterbrach die wissenschaftliche Entwicklung der Sozialhygiene für viele Jahre. Nach dem Tod von Grotjahn 1931 ließ das Ministerium für Wissenschaft, Kunst und Volksbildung das Ordinariat vakant, erteilte jedoch zur Fortsetzung des Unterrichtes einen Lehrauftrag an Benno Chajes (6). Bis zur Beurlaubung von Chajes 1933 durch das Ministerium verschwand der Stamm von 
Grotjahn-Schülern rasch im Zuge der politischen Entwicklung in Deutschland. Ein großer Teil der Teilnehmer waren Angehörige der jüdischen Religionsgemeinschaft bzw. politisch dem linken Lager zugehörig. Mit wachsendem Einfluss der Nationalsozialisten wurden viele von innen verhaftet, ermordet oder ins Exil getrieben. 1933 wurde Franz Schütz zum Außerordentlichen Professor für Sozialhygiene sowie zum Leiter des Sozialhygienischen Seminars ernannt und übernahm bis 1940 die Lehrveranstaltungen. Ebenfalls 1933 wurde Fritz Lenz zum Ordinarius für Rassenhygiene ernannt und richtete in den Räumen des Sozialhygienischen Seminars das Institut für Rassenhygiene ein. Er übernahm auch die Abteilung für Rassenhygiene des "Kaiser Wilhelm Institut für Anthropologie" (KWIA). Die Rassenhygiene wurde mit der Machtübernahme durch die Nationalsozialisten zur Leitwissenschaft. Ab 1938 war die Rassenhygiene Pflichtfach und ab 1941 endgültig als Unterrichtsfach an deutschen Hochschulen anerkannt.

Lenz gehörte darüber hinaus zum Sachverständigenausschuss für Bevölkerungs- und Rassenpolitik im Reichsministerium des Inneren und war 1940 an dem "Gesetz zur Sterbehilfe bei Leistungsunfähigen und Gemeinschaftsfremden" an der Formulierung des "Generalplan Ost“ beteiligt (7).

Mit dem Gesetz zur Wiederherstellung des Berufsbeamtentums vom 7. April 1933 und dem "Deutschen Beamtengesetzes" 1937 konnten Beamten aus politischen oder rassischen Gründen entlassen werden (Reichsgesetzblatt 1, Berlin 1933; 5). Als Folge wurde bis zum Jahre 1938 ein Drittel aller Lehrkräfte ausgewechselt, bis 1945 bereits $45 \%$, die fast alle in der Folgezeit zur Auswanderung gezwungen wurden $(8,9)$. Unter diesen Emigranten befanden sich einige der berühmtesten Wissenschaftler des 20. Jahrhunderts, darunter 24 Nobelpreisträger.

Im folgenden werden die Lebensläufe deutscher Sozialmediziner nach ihrer Emigration in die USA anhand der Einzelschicksale von Franz Goldmann, Alfred Korach, Miron Kantorowicz und Georg Wollf dargestellt. Diese Wissenschaftler waren Schüler bzw. Mitarbeiter von Alfred Grotjahn und emigrierten in den dreißiger Jahren als Angehörige der jüdischen Religion sowie als Mitglieder sozialdemokratischer oder kommunistischer Parteien aus dem nationalsozialistischen Deutschland in die USA aus. Sie konnten nach der Emigration ihre Fachkenntnisse nutzen und waren in Public Health Bereichen tätig. 
Die Suche nach Primärquellen erfolgte u.a. in folgenden Archiven: 1. Archiv der Humboldt-Universität Berlin, 2. Bundesarchiv in Koblenz, 3. National Institute of Health in Washington, 4. NARA-Archive in Washington, 5. Alan-Mason-Chesney-MedicalArchives in Baltimore. Sekundärquellen fanden sich in verschiedenen deutschen, amerikanischen und englischen Bibliotheken. Darüber hinaus wurde auf vorhandene Biografien zurückgegriffen $(4,14)$.

\section{Franz Goldmann}

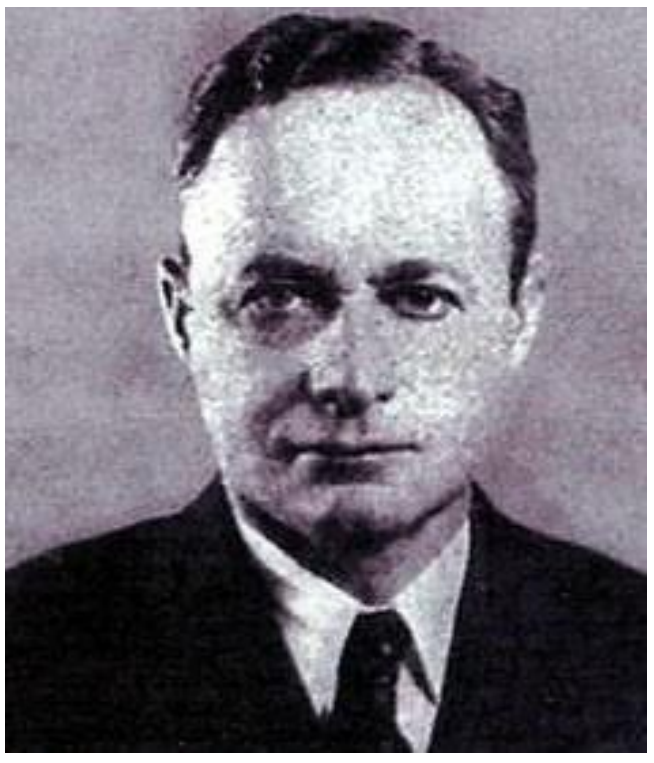

Abb. 2: Franz Goldmann; M. E. Grenader Department of Special Collections and Archives; University at Albany, State University of New York.

Franz Goldmann, geb. 1895 in WuppertalElberfeld als Sohn eines Pharmazeuten, studierte von 1913-1920 in Heidelberg und Berlin Medizin, wo er auch an Grotjahns „Sozialhygienischen Übungen“ teilnahm. 1932 habilitierte er unter dessen Betreuung „Über die gesundheitspolitisch wichtige Leistung der deutschen Krankenhäuser" und wirkte als Privatdozent an der Berliner Universität. 1933 verließ er Deutschland nach Inkrafttreten des Gesetzes zur Wiederherstellung des Berufsbeamtentums aus Angst vor politischer Verfolgung. 1937 emigrierte er nach einer Odyssee durch verschiedene Länder in die USA. Im gleichen Jahr begann er mit seiner Arbeit als Fellowship am „Dept. of Public Health“ an der Yale University, wo er eine neue Unterrichtsmethode etablierte, bei der den Studenten der Besuch von Vorlesungen, praktischen Übungen und die Umsetzung selbständig durchgeführter Feldstudien abverlangt wurde. Das neue Unterrichtsfach, ab 1941 unter dem Namen „Medical Care in Modern Society. Social and Economic Aspects in Medicine" löste im Bereich der Wissenschaft eine rege Debatte aus. Goldmann nahm in diesem Programm eine aktuelle Analyse des amerikanischen Gesundheitssystems vor und überprüfte dessen Finanzierbarkeit. Er deckte Mängel der freiwilligen Versicherungen auf, verwies auf die Vorteile einer gesetzlichen Krankenversicherung und stellte unter Einbeziehung der Argumente der 
Gegnerschaft der gesetzlichen Krankenversicherung die aktuelle Gesetzeslage zur Diskussion. Goldmann unterrichtete zwischen 1941 und 1942 in Yale, als Gastdozent an der "Columbia University, am "Dept. of Social Science“ und am "Connecticut College" in New London. Er wirkte als Berater des "Bureau of Research and Statistics“, des "Social Security Board“ u. a. in Washington. Er hielt Vorträge an der "Birth Control League of Connecticut" (10,11). 1947 folgte Goldmann einem Ruf an die Harvard University, wo er den Unterricht im Bereich Public Health ausbaute.

Franz Goldmann hatte in Deutschland die Zeit der Weltwirtschaftskrise und der Weimarer Republik erlebt, analysiert und sich für gesellschaftliche und politische Maßnahmen wie die Verankerung einer Familienversicherung in die Krankenversicherung eingesetzt. In den USA war er einer der ersten, der eine komplexe Analyse des Gesundheitswesen nahm. Er entwickelte in seinen Publikationen weitreichende Vorschläge, in denen er auf eine Zusammenarbeit der Institutionen und den Abbau von Egoismen verschiedener Beteiligter drängte. Dass die Etablierung einer allgemeinen gesetzlichen Pflichtversicherung in den USA nicht gelang, interpretierte er als Ausdruck des Scheiterns von Amerika in der Pflicht zur sozialen Verantwortung (10).

\section{Alfred Korach}

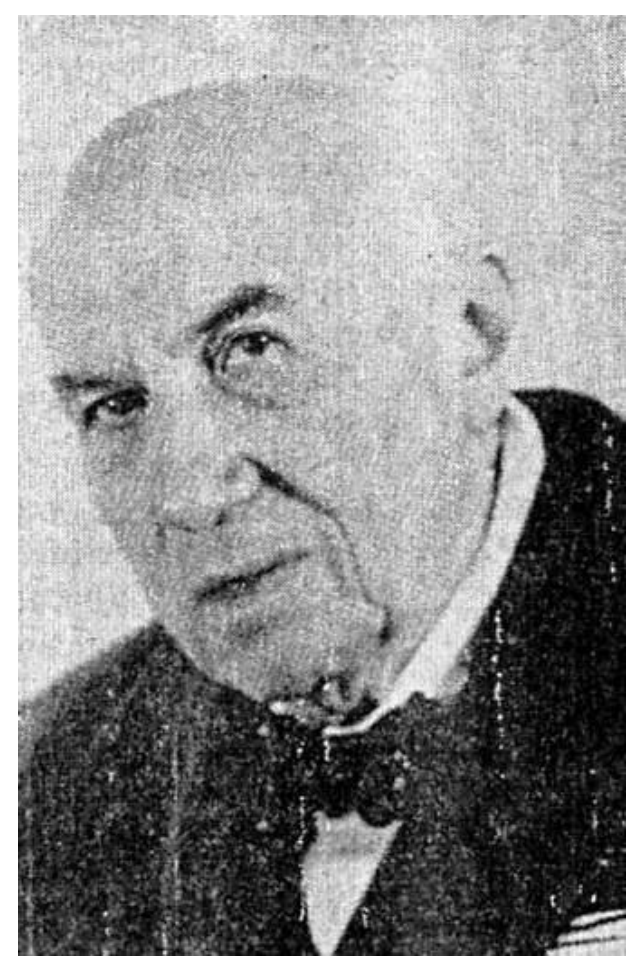

Abb. 3: Alfred Korach; M. E. Grenader Department of Special Collections and Archives; University at Albany, State University of New York.

Alfred Korach wurde 1893 in Königsberg als Sohn eines jüdischen Kaufmanns geboren. $\mathrm{Er}$ studierte von 1911 bis 1919 Medizin und Volkswissenschaft in München, Königsberg und Berlin, mit Schwerpunkten im Bereich der Sozialhygiene. Nach der Promotion 1920 besuchte er von 1921 bis 1922 die Grotjahnschen Sozialhygienischen Übungen. 1933 floh Alfred Korach mit seiner späteren Ehefrau Käthe Meyer nach England und von 
dort 1936 weiter nach Stockholm und 1937 in die USA. 1939 wurden Käthe und Alfred Korach vom Deutschen Reich ausgebürgert. Korach begann seine Lehrtätigkeit am „Massachusetts Institut" in Cambridge und wechselte 1939 als „Assistent Professor" für Öffentliches Gesundheitswesen an die Universität von Cincinnati/Ohio. 1939 wurde er amerikanischer Staatsbürger und änderte daraufhin seinen Familiennamen zu George. Nach seiner Emeritierung im Jahre 1963 siedelte er mit seiner Frau ins schweizerische Männendorf, wo er 1979 starb (12).

Im Oktober 1942 erschien das Medical Bulletin der University of Cincinnati unter dem Titel "Extra Mural Teaching of Preventive Medicine and Public Health" unter Korachs Herausgeberschaft, welches der Vermittlung von Kenntnissen über soziale und ökonomische Einflussgrößen auf die Gesundheit der Menschen diente. Zudem findet sich der Hinweis "Alfred Korach MD, Lecturer in Preventive Medicine, University of Cincinnati, College of Medicine".

„Extra Mural Teaching" bedeutet in etwa: außeruniversitäre Ausbildung. Korach unterrichtete in den Jahren 1939 bis 1942 im Schnitt 72 Studenten pro Jahr auf dem Gebiet der Feldforschung, wo er zeigen wollte, dass die Medizin weitaus komplexer ist als die beengte Sichtweise von Diagnose und Behandlung einer Krankheit. $\mathrm{Er}$ verdeutlichte, dass Krankheiten von vielen Faktoren beeinflusst werden und Prävention daher in viele Richtungen zielen müsse. Zudem gab es starke Verzweigungen in die Bereiche der Soziologie, Psychologie, Recht und Ökonomie. Soziale und ökonomische Faktoren, Probleme der Epidemiologie und Hygiene, Umwelteinflüsse sowie die verschiedenartigen Einflüsse der Public-Health-Tendenzen wurden untersucht (13). Korachs Bericht „Extral Mural Teaching of Preventive Medicine and Public Health" beschreibt eine gelungene Umsetzung vieler theoretischer Forderungen namhafter amerikanischer Wissenschaftler in Public Health aus dieser Zeit. Korach führt in der Darlegung des aktuellen Meinungsbildes vor allem amerikanische Forscher auf (13). Sein Lehrkonzept war die Quintessenz der damaligen Entwicklungen auf dem Gebiet der Gesundheitswissenschaft in den USA. 


\section{Miron Kantorowicz}

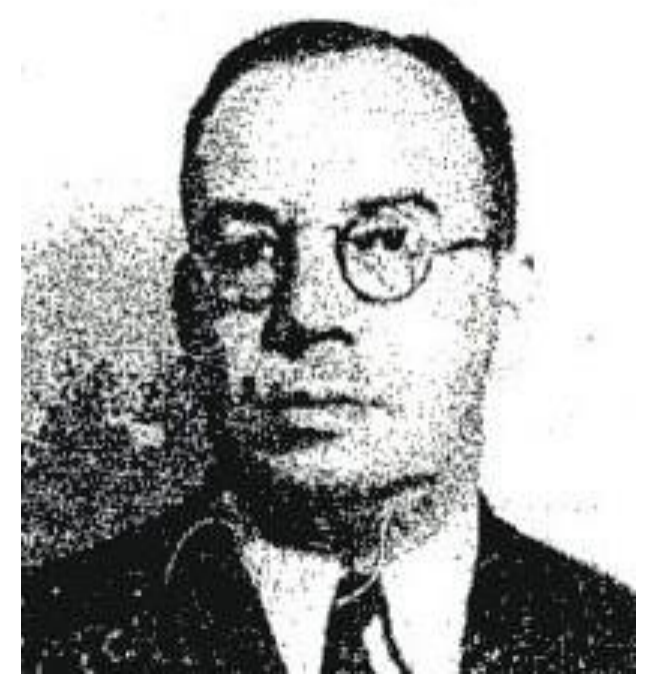

Abb. 4: Miron Kantorowicz; U.S. Citizenship and Immigration Services / COW 2006000697.

Miron Kantorowicz, geb. 1895 als Sohn eines Kaufmanns und Fabrikbesitzers in Minsk, studierte ab 1915 an der Universität Petrograd und ab 1917 in Moskau Jura und emigrierte nach der Oktoberrevolution im Jahre 1917 nach Berlin, wo er zwischen 1920 und 1930 Ökonomie, Geschichte und Philosophie studierte. 1930 folgte die Promotion zum Thema „Die Tuberkulosesterblichkeit und ihre sozialen Ursachen." Von 1929 bis 1933 arbeitete er als Bibliothekar von Alfred Grotjahn und war damit dessen einziger hauptberuflicher Mitarbeiter (14). Nach dem Tode Grotjahns hielt Kantorowicz in den „Sozialhygienischen Übungen“ einen Vortrag über dessen wissenschaftliches Werk und verfasste einige Artikel, in denen er die Theorien seines Lehrers darlegte (14). Nach Übernahme der sozialhygienischen Lehre durch Benno Chajes und später Franz Schütz wurde Kantorowicz im Sommer 1933 entlassen. Bis 1934 verblieb er in Berlin und recherchierte über Biometrie, Anthropologie und Eugenik, versuchte dabei aber im Ausland eine Anstellung zu finden. In dieser Zeit erhielt Kantorowicz ein Stipendium bei der „Jewish Health Organization of Great Britain" und wanderte noch 1934 nach England aus. Er fand langfristig jedoch in England keine Möglichkeit, sich weiter sozialhygienisch zu betätigen und siedelte deshalb 1938 in die USA über. 1940 wurde er als „Research Fellow“ im Bereich „Biostatistics“ von der "Milbank Memorial Foundation“ in New York eingesetzt. 1942 wechselte er an die „American University“ in Washington, wo er unter Leitung des Demographen Frank Lorimer, den er seit 1939 persönlich kannte, arbeitete. Lorimer veröffentlichte 1946 seinen umfassenden Bericht zur Geschichte der Bevölkerung der Sowjetunion. Während der Zeit zwischen der Beendigung seiner Arbeit an der „American University“ und dem Beginn seiner Arbeit als „Research Analyst“ am „Dept. of State“ veröffentlichte Kantorowicz den Artikel „Russia's growing population“ für die „American Academy of Political and Social Sience“ (14). 
1945 erlangte er die amerikanische Staatsbürgerschaft und änderte seinen Namen zu Myron Gordon (15). Von 1945 bis 1946 arbeitete er als "Chief Population and Manpower sect. Dept. State“, von 1946 bis 1954 als "head of the Slavic and Balkan Unit" an der Abt. für Präventivmedizin des "Office Surgeon Generale des U.S. Dept. Army“ und ab 1954 als Leiter der "East European Section, Med. Information und Intel. Div." Er blieb bis zu seiner Berentung 1963 im Dienst der Armee. Kantorowicz verfasste in seiner Funktion als Leiter der "East European Section" des "Office of the Surgeon General U.S. Army" 1955 eine Analyse des russischen Gesundheitssystems mit dem Titel "Standard of Living and Public Health in the USSR" (16).

Kantorowicz/Gordon hatte eine umfassende geisteswissenschaftliche Ausbildung und breit gefächertes Wissen in Geschichte, Ökonomie, Medizin und Sozialhygiene sowie Bevölkerungsstatistik. Diese Kenntnisse kamen inm in seiner Tätigkeit als „Research Fellow" am "Milbank Memorial Found“ in New York zugute. Für spätere Tätigkeiten, wie die Arbeit an der "American University“ in Washington oder seine Laufbahn am „Office Surgeon General U.S. Army“, war er mit seiner russischen Herkunft und als mehrsprachiger und breit gefächert gebildeter Wissenschaftler sehr geeignet. Kantorowicz war prädestiniert für die Übernahme administrativer Aufgaben, wie er sie als Leiter verschiedener Abteilungen innehatte. Seine Veröffentlichung „Standard of Living and Public Health in the USSR" aus dem Jahre 1955 bestätigt diese Rolle als Experte aus Osteuropa mit einem fundierten Wissen zu allen Aspekten des Public Health. Kantorowicz hinterließ fast keine Publikationen und auch sonst finden sich kaum noch Spuren seines Wirkens in den Bibliotheken oder Archiven der USA. Das Werk Grotjahns blieb bis zuletzt eines seiner großen Interessengebiete, wie ein Nachtrag aus dem Jahre 1975 zeigt. Es steht wohl außer Frage, dass er sein epidemiologisches Rüstzeug während seiner Studienzeit in Deutschland und durch seine Tätigkeit als Bibliothekar Grotjahns erworben hatte. 


\section{Georg Wollf}

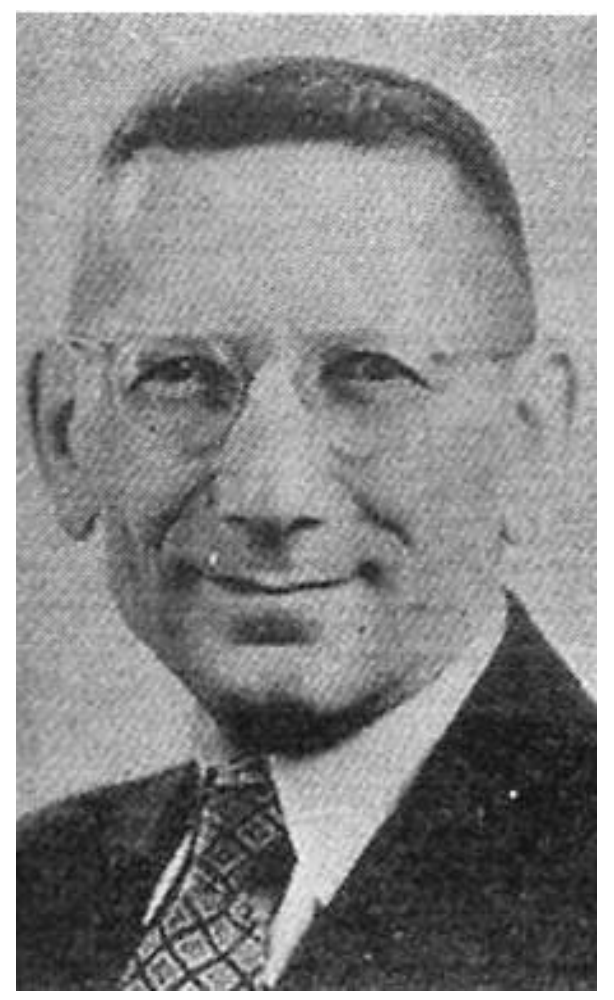

Abb. 5: Georg Wollf; U. S. Citizenship and Immigration Services / COW 2006000698.

Georg Wollf wurde 1886 in Sangerhausen als Sohn eines jüdischen Kaufmanns geboren. $\mathrm{Er}$ begann nach einer kaufmännischen Ausbildung 1906 mit dem Medizinstudium in Berlin und wurde 1911 approbiert. Im gleichen Jahr folgte seine Promotion und der Beginn seiner ärztlichen Laufbahn zunächst als Assistent an den Berliner Kinderkliniken sowie am Virchow-Klinikum in Berlin.

Zwischen 1920 und 1924 besuchte er die "Sozialhygienischen Übungen" von Alfred Grotjahn und habilitierte unter dessen Supervision im Jahre 1930. Zwischen 1927 und 1933 war er der Leiter der Abteilung Medizinalstatistik des Hauptgesundheitsamtes der Stadt Berlin. Am 7. April 1933 entzog inm das Ministerium für Wissenschaft, Kunst und Volksbildung wegen $\S 4$ des Gesetzes zur Wiederherstellung des Berufsbeamtentums aus rassischen Gründen die Lehrbefugnis (17).

1937 emigrierte Wollf in die USA und begann dort mit einer Lehr- und Forschungstätigkeit an der "Johns Hopkins University“ in Baltimore im Bereich des Public Health mit Unterstützung eines Stipendiums der „Milbank Memorial Foundation“. Ab 1941 wirkte er an der "Division of Public Health Methods” in Hagerstown am "National Institute of Health", NIH in Washington. Ab 1943 arbeitete er an der "Carnegie Institution" an der Abteilung für Genforschung in Cold Springs Harbor, Long Island in New York, ab 1944 auch gelegentlich für das „U.S. Children's Bureau“ an der Abteilung für Statistische Forschung und als "Research Fellow" des amerikanischen Arbeitsministeriums des Pentagon im „Aviation Bureau“. Von 1949 bis 1952 war Wollf für die „Medical Intellegence Branch of the Army Surgeon General's Office" tätig. Im September des Jahres 1952 war er Leiter der Abteilung für Biometrie der "Medical Devision, Civil Aeronautica Administration, CAA. Wollf starb 1952 an einer Herzattacke in Washington (18). 
Georg Wollf veröffentlichte im Auftrag des U.S. Childrens Bureau die Analyse großer amerikanischer Studien, die sich mit den Ursachen von Mortalität und den Auswirkungen von Krankheiten befassten. Seine Arbeit stellte die sozialen Ursachen von Krankheiten, die Unterschiede in der Prävalenz zwischen unterschiedlichen Regionen der USA, zwischen Rassezugehörigkeit, Alter und Geschlecht dar und leitete entsprechende Empfehlungen zur Prävention ab. 1952 veröffentliche Wollf unter dem Titel "The Social Pathology as a Medical Science“, eine Diskussion zum Thema "Soziale Pathologie als eine medizinische Wissenschaft", die eine gesamte Analyse der amerikanischen Entwicklung des Public Health beinhaltete und das Erbe Grotjahns aufgriff. Er kritisierte darin das angloamerikanische Defizit an einer systematischen Präsentation von sozialpathologischen Faktoren wie es in Deutschland unter Grotjahn entwickelt wurde und appellierte in seinen abschließenden Worten: „The investigation into the social etiology of disease, which is the essence of social medicine, is a necessity in any industrialized economy of our age, be it capitalism and free enterprise, or socialism and planned economy....The social pathologist and medical statistician as well as the medical planner and administrator must have not only a good knowledge of basic medical and social facts, of statistics and their limitations, he must also have a 'keen interest in humanity'." (19).

\section{Fazit}

Die vorliegenden biografischen Informationen zu Franz Goldmann, Alfred Korach, Miron Kantorowicz und Georg Wollf verdeutlichen eine relevante Mitarbeit der emigrierten deutschen Sozialmediziner in verschiedenen Bereichen des Gesundheitssystems in den USA. Auch wenn die biografischen Details nicht lückenlos bekannt sind, verdeutlichen doch die hinterlassenen Publikationen, dass ihre Arbeit im Rahmen von Public Health Konzepten erfolgte. Die deutschen Sozialmediziner fanden in den USA in bereits bestehenden Strukturen neue Aufgaben. Sie konnten mit ihrer wissenschaftlichen Arbeitsweise, breit gefächerten Kenntnissen auf dem Gebiet der Sozialhygiene und Erfahrungen aus Grotjahns „Sozialhygienischen Übungen“ wichtige Einzelbeiträge leisten. Gesellschaftssystem und Mentalität der USA haben den beruflichen Hintergrund und die Emigrationserfahrungen ehemaliger Sozialmediziner der Charité offenbar positiv gewertet. 


\section{Literatur}

1. Schagen U, Schleiermacher S. 100 Jahre soziale Medizin in Deutschland. Gesundheitswesen. 2006;68:85-93

2. Schallmayer W. Sozialhygiene und Eugenik. Z Sozialmed 1914;V.

3. Ferdinand U. Der Weg Alfred Grotjahns (1869-1931) zum "faustischen Pakt" in seinem Projekt der Sozialen Hygiene. Gesundheitswesen 2007;69:158-164

4. Grotjahn A. Erlebtes und Erstrebtes: Erinnerungen eines sozialistischen Arztes/Alfred Grotjahn. Berlin: Kommissions-Verlag. 1932: 284

5. $\quad$ Kaspari C. Alfred Grotjahn (1869-1931) - Leben und Werk. Dissertation. Bonn. 1989

6. Weder H. Sozialhygiene und pragmatische Gesundheitspolitik in der Weimarer Republik am Beispiel des Sozial- und Gewerbehygienikers Benno Chajes (1880-1938). In: Winau R, Bleker J (Hrsg.) Abhandlungen zur Geschichte der Medizin und Naturwissenschaft, Heft 87. Husum: Matthisen Verlag. 2000.

7. Schleiermacher S. Rassenhygiene und Rassenanthropologie an der Universität Berlin. In: Jahr C. (Hrsg.) Die Berliner Universität in der NS-Zeit, Band 1, Strukturen und Personen. München: Franz Steiner Verlag. 2005

8. Samuel RH, Hinton T. Education and Society in Modern Germany. London. 1949

9. Pross H. Die deutsche akademische Emigration nach den Vereinigten Staaten 1933-1941. Berlin: Duncker \& Humblot. 1955

10. Antoni C. Sozialhygiene und Public Health: Franz Goldmann (1895-1970). In: Winau R, MüllerDietz H (Hrsg.) Abhandlungen zur Geschichte der Medizin und der Naturwissenschaften, Heft 78. Husum: Matthiesen Verlag. 1997

11. Schneck P. Sozialhygiene und Rassenhygiene in Berlin: Die Schüler Alfred Grotjahns und ihr Schicksal unter dem NS-Regime. In: Fischer W, Hierholzer K, Hubenstorf M, Walther PT, Winau R (Hrsg.) Exodus von Wissenschaften aus Berlin: Fragestellungen - Ergebnisse - Desiderate; Entwicklungen vor und nach 1933. Berlin: Walter de Gruyter. 1994

12. Nachruf Prof. Dr. Alfred K. George; M.E. Grenander Department of Special Collections and Archives; University at Albany, State University of New York.

13. Korach A. Extra-Mural Teaching of Preventive Medicine and Public Health. Medical Bulletin. Cincinnati: University of Cincinnati. 1942

14. Tutzke D. Alfred Grotjahns Bibliothekar. Schr Ges Hyg 1972;18: 506-508

15. Biographical Directory of the American Public Health Association, New York, 1979 und U.S. Dept. of Homeland Security, Washington, Myron Gordon, März 2006

16. Gordon M (alias Kantorowicz, Miron). Standard of Living and Public health! in the U.S.S.R. by Myron Gordon; New York, 1955.

17. Schagen U, Schleiermacher S im Auftrag der Deutschen Gesellschaft für Sozialmedizin und Prävention (DGSMP) (Hrsg.) 100 Jahre Sozialhygiene, Sozialmedizin und Public Health in Deutschland; CD-Rom, Berlin 2005

18. Nachruf "Georg Wollf, CAA Medical Offficer, Dies", The Washington Post, pg. 22, Washington, 17. September 1952

19. Wollf G. Social Pathology as a Medical Science. Civil Aeronautics Administration (Hrsg.). Washington D.C.. 1952: 1581 